\title{
Role of transplantation in treatment of multiple myeloma in era of novel agents
}

\author{
Bartosz Małecki ${ }^{*}$, Lidia Gil, Dominik Dytfeld \\ Department of Hematology and Bone Marrow Transplantation, University of Medical Sciences in Poznan, Poland
}

\begin{abstract}
Multiple myeloma (MM) is a B-cell malignancy characterized by clonal proliferation of plasma cells. Despite the introduction of novel agents such as immunomodulatory drugs, proteasome inhibitors, and monoclonal antibodies, high-dose chemotherapy with autologous transplantation remains the primary treatment for patients with newly diagnosed multiple myeloma.

This review presents the results of clinical trials assessing the effectiveness and safety of various kinds of transplantation such as single, allogeneic, tandem and salvage. Nowadays, in the era of access to new therapies, the following questions should be asked: when is the best time to perform autologous transplantation? What is the significance of allogeneic or tandem transplantation? Is the use of a second or third salvage transplant justified? Will chimeric antigen receptor T-cell (CAR-T) therapy become a valuable therapeutic method in MM? In this article, we will try to answer these questions.
\end{abstract}

Key words: multiple myeloma, novel agents, transplantation, autologous, allogeneic, CAR-T

Acta Haematologica Polonica 2021; 52, 2: 77-84

\section{Introduction}

Multiple myeloma (MM) is a monoclonal plasma cell disorder characterized by the proliferation of malignant plasma cells in the bone marrow, the detection of monoclonal protein in the serum and/or urine, as well as the occurrence of secondary end-organ damage [1]. This disease accounts for $1 \%$ of all neoplasms overall, and approximately $10 \%$ of hematological malignancies. The incidence of MM in Europe is estimated at 4.5-6.0/100,000, the median age at diagnosis is 70 , and it is more common in men than in women.

The history of treatment for MM has changed since the introduction of high-dose chemotherapy with autologous stem cell transplantation (HDT/ASCT), and it has been improved by the advent of novel agents such as immunomodulatory drugs (IMiDs such as thalidomide, lenalidomide and pomalidomide), proteasome inhibitors (Pls such as bortezomib, carfilzomib, and ixazomib), and most recently monoclonal antibodies such as elotuzumab and daratumumab.

In this review, we will try to assess the current role of HDT/ASCT based on the results of studies.

\section{ASCT versus non-transplant-based strategies}

The first studies to compare the effectiveness of HDT/ASCT to standard-dose chemotherapy in multiple myeloma were conducted by the Intergroup Francophone du Myèlome (IFM) and the Medical Research Council (MRC). In both trials, high-dose chemotherapy and ASCT significantly prolonged progression-free survival (PFS) and overall survival (OS) compared to standard-dose chemotherapy without transplantation.

\footnotetext{
*Address for correspondence: Bartosz Małecki, Department of Hematology and Bone Marrow Transplantation, University of Medical Sciences in Poznan, Szamarzewskiego 84, 60-569 Poznan, Poland, phone: +48 6185493 83, e-mail: bartosz-malecki@wp.pl 
In a multicenter study, the Medical Research Council's Myeloma VII Trial, 407 patients with previously untreated multiple myeloma who were younger than 65 years received either conventional-dose combination chemotherapy (doxorubicin, carmustine, cyclophosphamide, melphalan) or intensive therapy (doxorubicin, vincristine, methylprednisolone, cyclophoshamide) with HDT (melphalan)/ASCT. Compared to standard therapy, intensive treatment increased median survival by almost 12 months: 54.1 months versus 42.3 months. The median duration of progression-free survival was 31.6 months in the intensive-therapy group compared to 19.6 months in the standard-therapy group. 206 deaths (89\%) were related to myeloma or toxicity of treatment. Multiple myeloma was a causal factor in more deaths in the standard-therapy group than in the intensive-therapy group (62\% vs. 49\%). Infection was reported in 68 patients (33\%), and was more frequent in the intensive-therapy group than in the standard-therapy group (37\% vs. 29\%) [2].

The IFM included 200 patients with untreated MM who were younger than 65 to receive either conventional-dose combination chemotherapy [VMCP (vincristine, melphalan, cyclophosphamide, prednisone), or BVAP (vincristine, carmustine, doxorubicin, prednisone)] or intensive therapy (both VMCP and BVAP) with HDT(melphalan)/ASCT. The probability of event-free survival for five years was $28 \%$ in the high-dose group and $10 \%$ in the conventional-dose group ( $p=0.01$ ). The overall estimated rate of five-year survival was $52 \%$ in the high-dose group and $12 \%$ in the conventional-dose group ( $p=0.03$ ). Treatment-related mortality (TRM) was similar in both groups [3].

At the time of these trials (published before 2010), the palette of available therapies for MM patients was limited and did not include novel agents as part of the initial treatment of patients with newly diagnosed multiple myeloma (NDMM).

A study comparing HDT/ASCT to new therapeutic regimens was conducted by Palumbo et al. [4]. 402 patients were enrolled, who after a lenalidomide and dexamethasone (Rd) induction were randomized to either two courses of high-dose melphalan $\left(200 \mathrm{mg} / \mathrm{m}^{2}\right)$ followed by ASCT, or six cycles of melphalan, prednisone and lenalidomide (MPR). Patients in the ASCT arm had significantly longer PFS (median: 43 vs. 22 months; $p<0.001$ ) and a higher 4 -year OS rate ( $82 \%$ vs. $65 \% ; p=0.02)$. Hematologic and nonhematologic adverse events were more frequent with high-dose melphalan than with MPR, but toxic effects were tolerable and did not affect the rate of early death or treatment discontinuation.

The next trial, conducted by Gay et al. [5], included 389 patients after induction with lenalidomide and dexamethasone $(\mathrm{Rd})$. After induction they were randomized to consolidation with either RCD regimen (lenalidomide, cyclophosphamide, dexamethasone) or two courses of high-dose melphalan/ASCT. They also randomized patients to maintenance with lenalidomide plus prednisone, or lenalidomide alone. PFS during consolidation was significantly shorter with chemotherapy plus lenalidomide compared to high-dose melphalan and ASCT (median 29 months vs. 43 months; $p<0.0001$ ) and there was a better 4-year OS rate $(73 \%$ vs. $86 \% ; p=0.004)$. Although HDT/ASCT induced more grade III-IV adverse events, no increase in serious adverse events or treatment-related deaths were noticed.

The phase III study of the European Myeloma Network (EMN02/H095) included an induction therapy with 3-4 cycles of VCD (bortezomib, cyclophosphamide and dexamethasone) followed by randomization between standard-dose therapy with VMP (bortezomib, melphalan and prednisone) versus high-dose therapy with melphalan at $200 \mathrm{mg} / \mathrm{m}^{2}$. The primary study endpoint was PFS. Median follow-up was 38 months: median PFS was not yet reached in the ASCT group and was 44 months in the VMP group; 3-year estimate of PFS was $64 \%$ vs. $57 \%$, respectively ( $p=0.002$ ), which represented a $24 \%$ reduced risk of progression or death in the ASCT group compared to the VMP group. $12 \%$ of deaths were probably related to treatment: $68 \%$ in the ASCT group and $32 \%$ in the VMP group, most frequently due to infections (21\%), cardiac events (16\%), and second primary malignancies (53\%) [6].

Attal et al. [7] conducted a phase III study to compare the efficacy and safety of a combination of lenalidomide, bortezomib and dexamethasone (RVD) alone versus RVD plus autologous transplantation in patients with newly diagnosed myeloma (NDMM). In this trial, PFS was significantly longer in the transplant versus the RVD group (median: 50 vs. 36 months; $p<0.001$ ). Transplantation versus RVD alone was associated with increased complete response (59\% vs. $48 \%$; $p=0.006$ ), but OS was similar in both arms (4-year survival of $81 \%$ in the transplant group vs. $82 \%$ in the RVD group). Hematologic and nonhematologic adverse events (grade III-IV) were more frequent in the transplant group versus the RVD group.

More and more single-arm studies are evaluating various combinations of novel agents as duplets and triplets in combination with ASCT. Recently published, the multicenter NCT01816971 phase II study has presented the relationship of ASCT with a carfilzomib-lenalidomide-dexamethasone (KRd) regimen for patients with NDMM. The patients received four cycles of $K R d$ in induction, $A S C T$, four cycles of $\mathrm{KRd}$ in consolidation, and 10 cycles of $\mathrm{KRd}$ in maintenance. There were high rates of SCR (stringent complete remission) and MRD-negative (minimal residual disease) at the end of $\mathrm{KRd}$ consolidation, and no treatment-related deaths were observed $[8,9]$.

All studies presented in this review comparing ASCT to non-transplant therapies in NDMM patients (Table I [2-7]) show a superiority of ASCT over the non-transplant approach in terms of high-quality response and PFS. 
Table I. Comparisons between autologous stem cell transplantation (ASCT) and non-transplant-based strategies (based on [2-7])

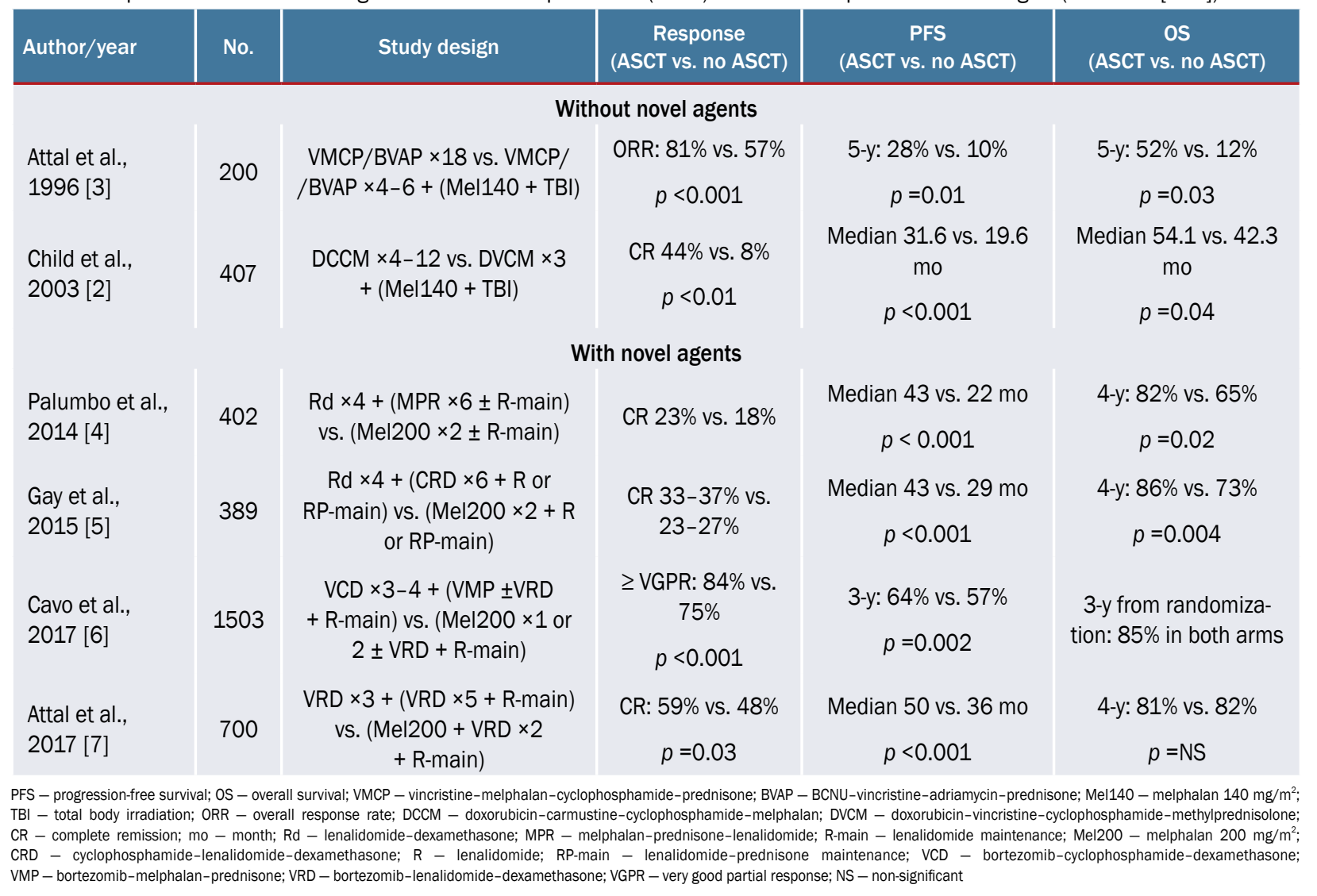

For these reasons, treatment with autologous stem cell transplantation should be considered in all patients with newly diagnosed multiple myeloma, despite the introduction of novel agents. Eligibility for this procedure should be based on performance status (Karnofsky index $\geq 90$ ) and hematopoietic cell transplantation-specific comorbidity in$\operatorname{dex}(\mathrm{HCT}-\mathrm{Cl}) \leq 2$. It seems that age is not itself a limitation for ASCT. The next challenge is to evaluate the necessity of HDT/ASCT when a monoclonal antibody such as daratumumab is added to an induction regimen combining an IMiD and a PI. To date, there has been no data from ongoing clinical trials comparing a non-transplant approach to a transplant approach including daratumumab. However, a few studies have estimated the efficacy and safety of this monoclonal antibody in combination with standard treatment for transplant-eligible patients (D-VTD vs. VTD - the CASSIOPEIA study and D-RVD vs. RVD - the GRIFFIN trial). The introduction of daratumumab improved depth of response and progression-free survival, with acceptable safety $[10,11]$.

\section{Single versus tandem ASCT}

A tandem transplant is defined as conducting a second procedure within 3-6 months of the first. The grounds for such a transplant are to achieve a deeper hematological response. In the era of new therapies available in the treatment of multiple myeloma, tandem transplantation has become less important [12].

In Attal's trial [13], patients with NDMM were randomized to one or two ASCTs after induction according to a VAD regimen (vincristine, doxorubicin, dexamethasone). In the group of patients after a single transplant, the therapy included melphalan $140 \mathrm{mg} / \mathrm{m}^{2}$ and TBI (8 Gy). In the group of patients who received tandem ASCT, patients received the first transplant after preparation of melphalan $140 \mathrm{mg} / \mathrm{m}^{2}$ alone. Melphalan $140 \mathrm{mg} / \mathrm{m}^{2}$ and TBI (8 Gy) were used before the second transplant. The probability of 7-year survival after diagnosis in the double transplant group compared to the single group was $42 \%$ versus $21 \%$ ( $p=0.01$ ). CR or VGPR was achieved by $49 \%$ of patients after a single transplant compared to $63 \%$ after tandem ASCT ( $p=0.01$ ), and the probability of relapse-free 7 -year survival after diagnosis was $13 \%$ in the single ASCT group and $23 \%$ in the tandem ASCT group ( $p<0.01$ ). In addition, the results indicate that double ASCT may be beneficial for patients who do not have a VGPR response after undergoing one ASCT. Indeed, the 7-year survival rate among these patients was $11 \%$ in the single ASCT group, and $43 \%$ in the double ASCT group $(p<0.001)$ [14]. Treatment-related deaths in the single-transplant group were $4 \%$, and in the double-transplant group $6 \%(p=0.40)$. 
In another clinical trial conducted by the Dutch-Belgian HOVON (Heamato Oncology Cooperative Group) group [15], patients were randomized after VAD induction chemotherapy to receive two cycles of non-myeloablative intermediate-dose melphalan $\left(70 \mathrm{mg} / \mathrm{m}^{2}\right.$ ) (single treatment) or the same regimen followed by cyclophosphamide $120 \mathrm{mg} / \mathrm{kg}$ intravenous plus total body irradiation (TBI) 9 Gy and autologous stem cell transplantation (double, intensive treatment). In this study, a significant difference was observed for PFS, but not for OS. The number of CRs was higher following a tandem procedure. Treatment-related mortality was $4 \%$ in the single treatment group, and $10 \%$ in the double, intensive arm.

In the EMN02/H095 phase III clinical trial described above [6], the efficacy of a single versus tandem transplant was compared at a later stage (415 patients were randomized). Patients who were eligible to tandem ASCT had a significantly higher 3 -year PFS rate (74\% vs. $62 \%$; $p=0.005)$ compared to those who underwent single ASCT.

In the phase III BMT-CTN 0702 STAMINA study, 758 patients with NDMM were randomized for induction, then subjected to firstly ASCT then either a second ASCT or RVD consolidation followed by lenalidomide maintenance. In contrast to other European studies, the investigators found no differences in terms of PFS (57\% vs. $57 \%$ ) or OS ( $86 \%$ vs. $82 \%$ ) between the two groups. Tandem ASCT did not have an advantage over single ASCT among high-risk patients [16].

In the modern era of novel agents, the value of a tandem ASCT is debatable. Tandem ASCT should be considered only for young patients of NDMM with high-risk disease characteristics and who did not achieve at least a VGPR after the first transplant. A carefully prepared clinical trial with modern therapies is needed to answer the questions about the role of tandem transplantation.

\section{Importance of salvage ASCT}

Salvage ASCT (SASCT) is defined as the administration of a second and subsequent ASCT at the time of relapse following a re-induction regimen [12]. According to the recent consensus from the American Society of Blood and Marrow Transplantation, the European Society for Blood and Marrow Transplantation, and the International Myeloma Working Group, sASCT should be considered in all patients with an initial duration of remission of $>18$ months following upfront ASCT [17]. However, in practice, the role and timing of SASCT varies between transplantation centers.

Several studies have evaluated the role of SASCT in the relapse of disease. They demonstrated that ASCT for a second, or even a third, time is an effective treatment option for patients who have previously undergone ASCT.

A prospective evaluation of SASCT was conducted by The Myeloma $X$ trial [18]. Patients were randomized to
SASCT or cyclophosphamide after a bortezomib-based re-induction at relapse. This clinical trial showed no OS advantage. The sASCT group had a significant improvement in PFS (19 vs. 11 months, $p<0.0001$ ) as well as in time to second objective disease progression - PFS2 (67 vs. 35 months, $p<0.0001)$. After progression, 20 patients $(85,27 \%)$ in the cyclophosphamide group underwent post-protocol salvage ASCT as third or fourth-line treatment. The PFS2 and OS in cyclophosphamide group split by subsequent-line salvage ASCT were not significantly different ( $p=0.269$ and $p=0.139$ ). The authors of this study concluded that patients derive the greatest benefits from consolidation of $\mathrm{SA}$ SCT immediately after first reinduction therapy at relapse. Delaying salvage ASCT to third-line or later may not confer the same degree of advantage.

The next prospective phase III multicenter trial, ReLApsE [19], included randomized patients with relapsed $\mathrm{MM}$ to receive either re-induction with lenalidomide-dexamethasone (Rd) followed by salvage ASCT and lenalidomide maintenance or Rd continuously. This was the first randomized clinical trial to compare salvage ASCT to treatment based on novel agents. This study showed a trend towards superior PFS (23.3 vs. 20.1 months; $p=0.09$ ) and significantly superior OS (NR vs. 57 months; $p=0.046$ ) in the arm with sASCT.

Garderet et al. [20] assessed retrospectively the outcome of salvage third ASCT in patients with relapsed MM. They analyzed 570 patients who had a third ASCT between 1997 and 2010. 482 patients underwent tandem ASCT and a third ASCT at first relapse (the AARA group) and 88 patients underwent an upfront ASCT with second and third transplantations after subsequent relapses (the ARARA group). Median follow-up after salvage third ASCT was 61 months in AARA and 48 months in ARARA, median PFS was 13 and 8 months, and median OS was 33 and 15 months. According to the relapse-free interval (RFI) from the second ASCT, if the RFI was < 18 months, then the median OS after the third ASCT was 17 months; if the RFI was $\geq 36$ months, then the median OS was 64 months in the AARA group $(p<0.001)$. In the ARARA group, if the RFI was $<6$ months, median OS after the third ASCT was seven months, 13 months if the RFI was $6-18$ months, and 27 months if the RFI was $\geq 18$ months ( $p<0.001)$.

In all the studies presented above, salvage ASCT was safe, with an expected increase in hematological and gastrointestinal toxicity but without TRM in patients up to the age of 75 .

These studies have demonstrated that SASCT is a safe and effective treatment option for patients with relapsed/ /refractory multiple myeloma (RRMM). It is important to carefully select patients who might benefit from SASCT. It should be used for patients with prolonged remission after a first or second ASCT, good general condition, and HCT-Cl below 3 (low-, intermediate-risk). In the future, 
Table II. Prospective trials comparing single or double autologous stem cell transplantation (ASCT) with ASCT followed by allogeneic stem cell transplantation (allo-SCT) (based on [22-25])

\begin{tabular}{|c|c|c|c|c|c|c|}
\hline Author/year & Treatment & No. allo vs. auto & Response CR (\%) & EFS/PFS & os & Comments \\
\hline $\begin{array}{l}\text { Garban et al., } \\
2006 \text { [22] }\end{array}$ & $\begin{array}{c}\text { Auto-allo (RIC) } \\
\text { vs. auto-auto }\end{array}$ & 65 vs. 219 & 62 vs. 51 & $\begin{array}{c}\text { Median EFS, } \\
31.7 \text { vs. } 35 \\
\text { mo }\end{array}$ & $\begin{array}{l}\text { Median OS, } \\
35 \text { vs. } 47.2 \\
\text { mo }\end{array}$ & $\begin{array}{c}\text { No differences in PFS } \\
\text { or OS }\end{array}$ \\
\hline $\begin{array}{l}\text { Rosiñol et al., } \\
2008 \text { [23] }\end{array}$ & $\begin{array}{l}\text { Allo vs. auto- } \\
\text {-auto }\end{array}$ & 25 vs. 85 & 40 vs. 11 & $\begin{array}{c}\text { Median PFS, } \\
\text { not reached } \\
\text { vs. } 31 \mathrm{mo}\end{array}$ & $\begin{array}{l}5 \text {-y } 0 \text { S, } 62 \% \\
\text { vs. } 60 \%\end{array}$ & $\begin{array}{l}\text { No differences in PFS } \\
\text { or OS }\end{array}$ \\
\hline $\begin{array}{l}\text { Giaccone et } \\
\text { al., } 2011 \text { [24] }\end{array}$ & $\begin{array}{l}\text { Auto-allo (TBI) } \\
\text { vs. auto-auto }\end{array}$ & 80 vs. 82 & 55 vs. 26 & $\begin{array}{c}\text { Median EFS, } \\
2.8 \text { vs. } 2.4 \\
\text { years }\end{array}$ & $\begin{array}{l}\text { Median OS, } \\
\text { NR vs. } 4.25 \\
\text { years }\end{array}$ & $\begin{array}{c}\text { Median } 7-y \text { follow-up, } \\
p=0.005 \text { for EFS and } \\
p=0.001 \text { OS favoring } \\
\text { auto-allo }\end{array}$ \\
\hline $\begin{array}{l}\text { Gahrton et } \\
\text { al., } 2013 \text { [25] }\end{array}$ & $\begin{array}{l}\text { Auto-allo vs. } \\
\text { auto-auto }\end{array}$ & 108 vs. 249 & 51 vs. 41 & $22 \%$ vs. $12 \%$ & $\begin{array}{c}49 \% \text { vs. } \\
36 \%\end{array}$ & $\begin{array}{c}\text { Median 8-y follow-up, } \\
p=0.027 \text { EFS, } p=0.030 \\
\text { for OS }\end{array}$ \\
\hline
\end{tabular}

the availability of novel agents may improve the response to a second or even a third ASCT, rather than impairing its usefulness, by enhancing the depth of response before ASCT.

\section{Role of allogeneic transplantation in treatment of multiple myeloma}

Despite increasing the possibilities and effectiveness of the treatment of multiple myeloma, it remains an incurable disease with a poor prognosis, especially in high-risk patients.

Allogeneic stem cell transplantation (allo-SCT) offers a potentially curative option due to a graft-versus-myeloma (GvM) effect, and may help achieve long-term PFS. However, currently allo-SCT remains a controversial treatment because of considerable toxicity, especially due to immunosuppression and severe infections, the risk of graft-versus-host disease (GvHD), and a potentially high non-relapse mortality (NRM) [21].

Greil et al. [21] conducted analysis of 109 patients with multiple myeloma who received allogeneic transplantation with reduced-intensity conditioning (RIC) between 2000 and 2016. Median patient age was 56 with a 1:1 proportion male:female. Most were treated in terms of individual salvage attempts due to relapsed/refractory disease after extensive earlier treatment. Only $50 \%$ of patients received regimens containing $\mathrm{PI}$, and $43 \%$ containing IMiDs. $92 \%$ of the cohort received prior auto-SCT, the majority of them as a single transplant; $24 \%$ received prior auto-SCT as a tandem transplant or with a second transplant in the case of relapse. After allo-SCT, 50\% of the cohort did not develop any sign of acute GvHD (aGvHD), in $25 \%$ only mild symptoms aGvHD grade I were found, and the remaining $25 \%$ were diagnosed with grades II-IV.
At the time of analysis, $42 \%$ of the patients were still alive: most patients died from PD with accompanying infections. The overall response rate was $70 \%$, the median OS was $39.2 \%$, and the median PFS was 14.2 months, with a median follow up of 71.5 months. Survival was significantly better in patients with response to previous therapies compared to patients with progressive disease (median OS: 65 vs. 11.5 months, $p=0.003$; median PFS 18.4 vs. 5.1 months, $p=0.001$ ). Apart from that, survival of patients transplanted in first-line was significantly prolonged compared to relapsed/refractory disease (median OS not reached vs. 21.6 months, $p<0.001$; median PFS 47.7 vs. 9.6 months, $p<0.001$ ). Treatment-related mortality was comparatively low, with a cumulative incidence of $12.4 \%$ over 10 years. The authors of this review suggest that the introduction of novel agents in combination with allogeneic transplantation in a group of selected patients with high risk of disease may significantly prolong their survival, and could even give the chance of a cure. However, these conclusions should await future prospective clinical trials.

The significance of allogenic transplantation was also analyzed in prospective studies comparing tandem ASCT versus ASCT-allo-SCT (Table II [22-25]). In all trials, these transplantations were a part of the initial therapy.

The IFM99-03/IFM99-04 trials [22] compared myeloma patients with high-risk myeloma (del13 by FISH or an elevated beta $_{2}$-microglobulin). All patients received melphalan $200 \mathrm{mg} / \mathrm{m}^{2}$ before single ASCT. The allo-SCT group received a reduced but myeloablative regimen of busulfan, fludarabine. The no-allo-SCT group received a second ASCT after melphalan $220 \mathrm{mg} / \mathrm{m}^{2}$. There were no differences in response rates (RR), PFS or OS. The 100-day mortality rate in these studies was $4.3 \%$, and the overall TRM rate was $10.9 \%$. 
Table III. B-cell maturation antigen (BCMA) chimeric antigen receptor T-cell (CAR-T) therapy trials [27]

\begin{tabular}{|c|c|c|c|c|c|c|}
\hline Variable & $\mathrm{NCl}$ & University of Pennsylvania & JNJ4528 Multicenter & \multicolumn{2}{|c|}{ Bluebird 2121 Multicenter } & Nanjing Legend \\
\hline $\begin{array}{l}\text { Authors/pre- } \\
\text { sentation }\end{array}$ & $\begin{array}{l}\text { Ali et al., } \\
\text { Blood } \\
\text { 2016; Brud- } \\
\text { no et al., } \\
\text { ASH } 2017\end{array}$ & $\begin{array}{l}\text { Cohen et al., ASH 2016; } \\
\text { Cohen et al., JCI } 2019\end{array}$ & $\begin{array}{c}\text { Berdeja et al., ASCO } \\
2020 \\
\text { Cartitude-1 trial } \\
\text { Phase Ib/II }\end{array}$ & $\begin{array}{c}\text { Berdeja et } \\
\text { al., ASH } \\
2017 ; \\
\text { Raje et } \\
\text { al., NEJM } \\
2019 \\
\text { Phase I }\end{array}$ & $\begin{array}{c}\text { KarMMa } \\
\text { trial } \\
\text { Phase II }\end{array}$ & $\begin{array}{l}\text { Fan et al., ASCO } \\
2017 \text {; Zhao et al., } \\
\text { J Hem Onc } 2018\end{array}$ \\
\hline$n$ & 24 & 25 & 29 & 33 & 128 & 57 \\
\hline Vector & $\begin{array}{l}\text { Retroviral } \\
\text { vector }\end{array}$ & Lentiviral vector & Lentiviral vector & Lentivi & vector & Lentiviral vector \\
\hline $\begin{array}{l}\text { Costimulatory } \\
\text { domain }\end{array}$ & $\mathrm{CD} 28$ & $4-1 B B$ & $4-1 B B$ & & & $4-1 B B$ \\
\hline $\begin{array}{l}\text { Activation do- } \\
\text { main }\end{array}$ & $\mathrm{CD} 3 \zeta$ & $\mathrm{CD} 3 \zeta$ & $\mathrm{CD} 3 \zeta$ & & & $\mathrm{CD} 3 \zeta$ \\
\hline Conditioning & Fludarabine & $\begin{array}{c}\text { With or without cyclopho- } \\
\text { sphamide }\end{array}$ & $\begin{array}{l}\text { Cyclophosphamide } \\
\text { +fludarabine }\end{array}$ & $\begin{array}{l}\text { Cyclophe } \\
\text { +flud }\end{array}$ & $\begin{array}{l}\text { hamide } \\
\text { bine }\end{array}$ & Cyclophosphamide \\
\hline $\begin{array}{l}\text { Prior lines } \\
\text { of treatment }\end{array}$ & 9 (median) & 7 (median) & 5 (median) & 8 (median) & $\geq 3$ & 3 (median) \\
\hline Response & $\begin{array}{l}\text { 81\% ORR; } \\
>\text { CR 13\% }\end{array}$ & $\begin{array}{l}\text { Cohort 1: 6/9 (1 sCR, } \\
2 \text { VGPR, } 2 \text { PR, } 1 \mathrm{MR}) \text {; } \\
\text { cohort 2: 2/5 (1 PR, } \\
1 \mathrm{MR}) ; \text { cohort 3: 5/6 } \\
\text { (1 CR, 3 PR, } 1 \mathrm{MR})\end{array}$ & $\begin{array}{c}100 \% \text { ORR; } 76 \% \\
\text { sCR; } 21 \% \text { VGPR; } \\
3 \% \text { PR }\end{array}$ & $\begin{array}{l}\text { ORR: } 85 \% ; \\
\text { >CR } 45 \%\end{array}$ & $\begin{array}{l}\text { ORR: } \\
\text { 73,4\% } \\
\text { CR/sCR: } \\
\text { 31.3\% }\end{array}$ & $\begin{array}{c}\text { ORR: } 88 \% ; 68 \% \\
\text { CR; } 5 \% \text { VGPR; } 14 \% \\
\text { PR }\end{array}$ \\
\hline PFS - median & $\begin{array}{c}\text { PFS: } 7,2 \\
\text { mo }\end{array}$ & $\begin{array}{l}\text { Cohort 1: } 2.2 \mathrm{mo} \\
\text { Cohort 2: } 1.9 \mathrm{mo} \\
\text { Cohort 3: } 4.2 \mathrm{mo}\end{array}$ & PFS: 6 mo & $\begin{array}{l}\text { PFS: } 11.8 \\
\text { mo }\end{array}$ & $\begin{array}{c}\text { PFS: } 8.6 \\
\text { mo }\end{array}$ & PFS: 15 mo \\
\hline
\end{tabular}

$\mathrm{NCl}$ - National Cancer Institute; ORR - overall response rate; CR - complete remission; SCR - stringent complete remission; VGPR - very good partial response; PR - partial response; $\mathrm{MR}$ - minimal response; PFS - progression-free survival; mo - month

The PETHEMA study [23] included only patients who achieved less than a near CR after a single ASCT. 25 patients with donors proceeded to allo-SCT after a reduced-intensity myeloablative regimen of fludarabine, melphalan, and they were compared to 85 patients without donors who received a second ASCT after a regimen of cyclophosphamide, etoposide and carmustine or melphalan $200 \mathrm{mg} / \mathrm{m}^{2}$. Statistically significant better CR rates for allo-SCT group without differences in PFS or OS and a trend toward a higher TRM with allo-RIC (16\% vs. 5\%; $p=0.09$ ) were found.

The next study was conducted by Giaccone et al. [24]. All patients received melphalan $200 \mathrm{mg} / \mathrm{m}^{2}$ before single ASCT and next allo-SCT after 2 Gy TBI or a second ASCT with 100-140 mg melphalan. This trial demonstrated CR rates of $55 \%$ versus $26 \%$, and improved PFS and OS rates (follow-up of 7 years) for the allo-SCT group. Treatment-related mortality in the allo-SCT group was $16 \%$, and $2 \%$ in the ASCT group.

A European trial (EBMT-NMAM2000) [25] compared 108 patients after a tandem ASCT-allo-SCT treatment (fludarabine, $2 \mathrm{~Gy} \mathrm{TBI}$ ) to 249 patients who received 1 or 2 ASCTs with melphalan $200 \mathrm{mg} / \mathrm{m}^{2}$. This trial also revealed a statistically better CR, PFS, and OS rates with follow-up at 8 years in favor of allo-SCT. Non-relapse mortality was $12 \%$ versus $2 \%$ at 24 months for the auto/allo group ( $p=0.003$ ).

To sum up the above prospective studies, only two of the four trials showed significantly better PFS and OS for the allo-SCT group. Therefore, despite biological rationale, the role of allo-SCT in the treatment of MM is limited. Based on current knowledge, this treatment method should be restricted to young, selected and motivated patients with high-risk multiple myeloma [26]. Today, as a result of various combinations and the wider use of novel agents in MM therapy, interest in allo-SCT has decreased significantly, and it remains mainly an investigational method.

\section{CAR-T cell therapy: future treatment of refractory/recurrent multiple myeloma?}

Despite the introduction of many new drugs that have dramatically changed the results of MM patients and significantly improved overall survival, many patients suffer from refractory and recurrent disease even after multiple lines of therapy $[27,28]$. 
Treatment options for these patients are particularly limited. Advances in cellular immunotherapy will probably lead to significant improvements in RRMM therapy. Chimeric antigen receptor (CAR) T-cell therapy represents a major advance in personalized malignance treatment. In this, a patient's own T cells are genetically engineered to express a synthetic receptor that binds to a tumor antigen. Currently, most clinical trials related to CARs in RRMM are directed against $\mathrm{B}$-cell maturation antigen (BCMA). BCMA is a member of the tumor necrosis factor superfamily of proteins that is primarily expressed by malignant and normal plasma cells and some mature B cells [29]. The first reports have shown promising results and safety profiles with even high risk features (Table III) [27]. There are other ongoing clinical trials also using CAR-T technology to target myeloma antigens such as CD138, CS1 glycoprotein antigen (SLAMF7), and immunoglobulin light chains. But these studies are still at an early stage [30].

\section{Conclusions}

Having been introduced approximately 30 years ago, ASCT remains the main therapeutic tool in the treatment of multiple myeloma for fit patients, despite the introduction of novel agents. It is an essential component of a complex treatment strategy that connects the use of new therapies in induction and consolidation or maintenance with high-dose chemotherapy/ASCT, and has an acceptable profile of toxic effects $[12,16]$.

In spite of many attempts to prove the importance of tandem and allogenic transplantation, their effectiveness has been questioned in many studies. These procedures should remain reserved for young, high-risk patients. At relapse, salvage ASCT presents an effective treatment option. In view of the availability of new drugs, we should consider the type and duration of response obtained after prior ASCT to select those patients who will benefit the most from SASCT.

Probably in the near future CAR-T therapy will become a significant method of treatment, especially for RRMM. However, it remains to be determined when will be the best time to incorporate this therapy in MM: as part of induction therapy, in the relapse, as an alternative to ASCT, or as an adjunct to ASCT?

To sum up, it is unclear whether CAR-T therapy will provide another weapon in an increasingly complex arsenal of multiple myeloma treatment options, or whether it might bring about a new standard of care for a disease that remains incurable.

\section{Authors' contributions}

All authors conceived the idea for this article, wrote the paper, edited and approved the final version.

\section{Conflict of interest}

None.

\section{Financial support}

None.

\section{Ethics}

The work described in this article has been carried out in accordance with The Code of Ethics of the World Medical Association (Declaration of Helsinki) for experiments involving humans; EU Directive 2010/63/EU for animal experiments; Uniform Requirements for Manuscripts submitted to Biomedical Journals.

\section{References}

1. Röllig C, Knop S, Bornhäuser M. Multiple myeloma. Lancet. 2015; 385(9983): 2197-2208, doi: 10.1016/s0140-6736(14)60493-1.

2. Child JA, Morgan GJ, Davies FE, et al. Medical Research Council Adult Leukaemia Working Party. High-dose chemotherapy with hematopoietic stem-cell rescue for multiple myeloma. N Engl J Med. 2003; 348(19): 1875-1883, doi: 10.1056/NEJMoa022340, indexed in Pubmed: 12736280.

3. Attal M, Harousseau JL, Stoppa AM, et al. A prospective, randomized trial of autologous bone marrow transplantation and chemotherapy in multiple myeloma. Intergroupe Français du Myélome. N Engl J Med. 1996; 335(2): 91-97, doi: 10.1056/NEJM199607113350204, indexed in Pubmed: 8649495.

4. Palumbo A, Cavallo F, Gay F, et al. Autologous transplantation and maintenance therapy in multiple myeloma. N Engl J Med. 2014; 371(10): 895-905, doi: 10.1056/nejmoa1402888.

5. Gay F, Oliva S, Petrucci MT, et al. Chemotherapy plus lenalidomide versus autologous transplantation, followed by lenalidomide plus prednisone versus lenalidomide maintenance, in patients with multiple myeloma: a randomised, multicentre, phase 3 trial. Lancet Oncol. 2015; 16(16): 1617-1629, doi: 10.1016/S1470-2045(15)00389-7, indexed in Pubmed: 26596670.

6. Cavo M, Hájek R, Pantani L, et al. Autologous stem cell transplantation versus bortezomib-melphalan-prednisone for newly diagnosed multiple myeloma: second interim analysis of the phase 3 EMN02/H095 Study. Blood. 2017; 130(Suppl 1): 397, doi: https://doi.org/10.1182/ /blood.V130.Suppl_1.397.397.

7. Attal M, Lauwers-Cances V, Hulin C, et al. IFM 2009 Study. Lenalidomide, bortezomib, and dexamethasone with transplantation for myeloma. N Engl J Med. 2017; 376(14): 1311-1320, doi: 10.1056/ /NEJMoa1611750, indexed in Pubmed: 28379796.

8. Jasielec JK, Kubicki T, Raje N, et al. Carfilzomib, lenalidomide, and dexamethasone plus transplant in newly diagnosed multiple myeloma. Blood. 2020; 136(22): 2513-2523, doi: 10.1182/blood.2020007522.

9. Vij R, Wang M, Jagannath $\mathrm{S}$, et al. An open-label, single-arm, phase 2 (PX-171-004) study of single-agent carfilzomib in bortezomib-naive patients with relapsed and/or refractory multiple myeloma. Blood. 2012; 119(24): 5661-5670, doi: 10.1182/blood-2012-03-414359, indexed in Pubmed: 22555973.

10. Moreau P, Attal M, Hulin C, et al. Bortezomib, thalidomide, and dexamethasone with or without daratumumab before and after autologous 
stem-cell transplantation for newly diagnosed multiple myeloma (CASSIOPEIA): a randomised, open-label, phase 3 study. Lancet. 2019; 394(10192): 29-38, doi: 10.1016/S0140-6736(19)31240-1, indexed in Pubmed: 31171419.

11. Voorhees PM, Kaufman JL, Laubach J, et al. Daratumumab, lenalidomide, bortezomib, and dexamethasone for transplant-eligible newly diagnosed multiple myeloma: the GRIFFIN trial. Blood. 2020; 136(8): 936-945, doi: 10.1182/blood.2020005288, indexed in Pubmed: 32325490.

12. Ntanasis-Stathopoulos I, Gavriatopoulou M, Kastritis E, et al. Multiple myeloma: role of autologous transplantation. Cancer Treat Rev. 2020; 82: 101929, doi: 10.1016/j.ctrv.2019.101929, indexed in Pubmed: 31770695 .

13. Attal M, Harousseau JL, Facon T, et al. InterGroupe Francophone du Myélome. Single versus double autologous stem-cell transplantation for multiple myeloma. N Engl J Med. 2003; 349(26): 2495-2502, doi: 10.1056/NEJMoa032290, indexed in Pubmed: 14695409.

14. Martino M, Recchia AG, Fedele R, et al. The role of tandem stem cell transplantation for multiple myeloma patients. Expert Opin Biol Ther. 2016; 16(4): 515-534, doi: 10.1517/14712598.2016.1136285, indexed in Pubmed: 26698133.

15. Sonneveld P, van der Holt B, Segeren CM, et al. Dutch-Belgian Hemato-Oncology Cooperative Group (HOVON). Intermediate-dose melphalan compared with myeloablative treatment in multiple myeloma: long-term follow-up of the Dutch Cooperative Group HOVON 24 trial. Haematologica. 2007; 92(7): 928-935, doi: 10.3324/haematol.11168, indexed in Pubmed: 17606443.

16. Mina R, Lonial $\mathrm{S}$. Is there still a role for stem cell transplantation in multiple myeloma? Cancer. 2019; 125(15): 2534-2543, doi: 10.1002/cncr.32060, indexed in Pubmed: 30985927.

17. Hagen PA, Stiff P. The role of salvage second autologous hematopoietic cell transplantation in relapsed multiple myeloma. Biol Blood Marrow Transplant. 2019; 25(3): e98-e9e107, doi: 10.1016/j. bbmt.2018.12.002, indexed in Pubmed: 30537552.

18. Cook G, Ashcroft AJ, Cairns DA, et al. National Cancer Research Institute Haemato-oncology Clinical Studies Group. The effect of salvage autologous stem-cell transplantation on overall survival in patients with relapsed multiple myeloma (final results from BSBMT/UKMF Myeloma X Relapse [Intensive]): a randomised, open-label, phase 3 trial. Lancet Haematol. 2016; 3(7): e340-e351, doi: 10.1016/S23523026(16)30049-7, indexed in Pubmed: 27374467.

19. Baertsch MA, Schlenzka J, Mai EK, et al. Rationale and design of the German-Speaking Myeloma Multicenter Group (GMMG) trial ReLApsE: a randomized, open, multicenter phase III trial of lenalidomide/dexamethasone versus lenalidomide/dexamethasone plus subsequent autologous stem cell transplantation and lenalidomide maintenance in patients with relapsed multiple myeloma. BMC Cancer. 2016; 16: 290, doi: 10.1186/s12885-016-2321-2, indexed in Pubmed: 27114074.
20. Garderet $L$, lacobelli $S$, Koster $L$, et al. Outcome of a salvage third autologous stem cell transplantation in multiple myeloma. Biol Blood Marrow Transplant. 2018; 24(7): 1372-1378, doi: 10.1016/j. bbmt.2018.01.035, indexed in Pubmed: 29408334.

21. Greil C, Engelhardt M, Ihorst G, et al. Allogeneic transplantation of multiple myeloma patients may allow long-term survival in carefully selected patients with acceptable toxicity and preserved quality of life. Haematologica. 2019; 104(2): 370-379, doi: 10.3324/haematol.2018.200881, indexed in Pubmed: 30237266.

22. Garban F, Attal M, Michallet M, et al. Prospective comparison of autologous stem cell transplantation followed by dose-reduced allograft (IFM99-03 trial) with tandem autologous stem cell transplantation (IFM99-04 trial) in high-risk de novo multiple myeloma. Blood. 2006; 107(9): 3474-3480, doi: 10.1182/blood-2005-09-3869, indexed in Pubmed: 16397129.

23. Rosiñol L, Pérez-Simón JA, Sureda A, et al. Programa para el Estudio y la Terapéutica de las Hemopatías Malignas y Grupo Español de Mieloma (PETHEMA/GEM). A prospective PETHEMA study of tandem autologous transplantation versus autograft followed by reduced-intensity conditioning allogeneic transplantation in newly diagnosed multiple myeloma. Blood. 2008; 112(9): 3591-3593, doi: 10.1182/ /blood-2008-02-141598, indexed in Pubmed: 18612103.

24. Giaccone L, Storer B, Patriarca F, et al. Long-term follow-up of a comparison of nonmyeloablative allografting with autografting for newly diagnosed myeloma. Blood. 2011; 117(24): 6721-6727, doi: 10.1182/blood-2011-03-339945, indexed in Pubmed: 21490341.

25. Gahrton G, lacobelli S, Björkstrand Bo, et al. EBMT Chronic Malignancies Working Party Plasma Cell Disorders Subcommittee. Autologous/reduced-intensity allogeneic stem cell transplantation vs autologous transplantation in multiple myeloma: long-term results of the EBMT-NMAM2000 study. Blood. 2013; 121(25): 5055-5063, doi: 10.1182/blood-2012-11-469452, indexed in Pubmed: 23482933.

26. Bensinger W. Allogeneic stem cell transplantation for multiple myeloma. Hematol Oncol Clin North Am. 2014; 28(5): 891-902, doi: 10.1016/j.hoc.2014.06.001, indexed in Pubmed: 25212888.

27. Feinberg D, Paul B, Kang $Y$. The promise of chimeric antigen receptor (CAR) T cell therapy in multiple myeloma. Cell Immunol. 2019; 345: 103964, doi: 10.1016/j.cellimm.2019.103964, indexed in Pubmed: 31492448.

28. Stirrups R. CAR T-cell therapy for relapsed or refractory multiple myeloma. Lancet Oncol. 2019; 20(6): e297, doi: 10.1016/s14702045(19)30288-8.

29. Lin Q, Zhao J, Song Y, et al. Recent updates on CAR T clinical trials for multiple myeloma. Mol Cancer. 2019; 18(1): 154, doi: 10.1186/ /s12943-019-1092-1, indexed in Pubmed: 31684964.

30. Dytfeld D. Chimeric antigen receptor $\mathrm{T}$ in the treatment of multiple myeloma - state of the art and future directions. Acta Haematol Pol. 2020; 51(3): 120-124, doi: 10.2478/ahp-2020-0023. 